\title{
The role of parenting, self-compassion and friendships in depressive symptoms among young people in the UK and China
}

\author{
Mengya Zhao ${ }^{1,2} \oplus \cdot$ Tamsin Ford $^{3} \cdot$ Peng Wang $^{4} \cdot$ Anke Karl $^{1}$
}

Accepted: 3 November 2021

(c) The Author(s) 2021

\begin{abstract}
Self-compassion, being kind to oneself in difficult times, is a way of relating to oneself that promotes better mental health, but little is known about how self-compassion affects interpersonal relationships. The current study examined the association between self-compassion and adolescent depressive symptoms from an interpersonal perspective in different cultural contexts. Adolescents $(N=422 / 570$, Mean age $=14.44 / 13.41$, UK/China) completed questionnaires about their perceptions of their parents' behaviour towards them, self-compassion, friendships and depressive symptoms. Structural equation modelling revealed that positive parenting was positively associated with higher self-compassion, positive friendship quality, and with fewer depressive symptoms in both samples. Additionally, we confirmed a negative association between self-compassion and depressive symptoms. The pathway from positive parenting to lower depressive symptoms via higher self-compassion was also corroborated in both cultures. Contrary to our hypothesis, positive quality of friendship was associated with higher depressive symptoms in both countries. Conflicts were associated with more depressive symptoms in the Chinese sample only. There was evidence of a negative association between self-compassion and conflicts in the Chinese sample only. Finally, the direct association between self-compassion and depressive symptoms was greater in the UK sample, whereas in the Chinese sample, interpersonal factors were more strongly associated with self-compassion and depressive symptoms. Our findings suggest that self-compassion may be a useful therapeutic target to improve social functioning and mental health among adolescents and that it may be necessary to account for cross-cultural differences in interpersonal factors when designing psychological interventions.
\end{abstract}

Keywords Adolescent depressive symptoms · Self-compassion · Parenting behaviour · Friendship $\cdot$ Cross culture

Mengya Zhao

m.zhao@exeter.ac.uk

Tamsin Ford

tjf52@medschl.cam.ac.uk

Peng Wang

pengsdnu@163.com

Anke Karl

a.karl@exeter.ac.uk

1 Psychology, College of Life and Environmental Sciences (CLES), University of Exeter, Exeter, UK

2 School of Psychology, University of Exeter, Washington Singer Laboratories, Perry Road, Exeter EX4 4QG, UK

3 Department of Psychiatry, University of Cambridge, Cambridge, UK

4 College of Psychology, Shandong Normal University, Jinan, China
The prevalence of adolescent depressive symptoms is increasing worldwide (Collishaw, 2015), and therefore, it is crucial to develop interventions to target adolescent depression and reduce the associated societal burden (Herrman et al., 2019). Adolescence is an important transition period characterised by puberty onset, interpersonal relationship changes (i.e., the relationships with parents and peers) and the development of self (Steinberg \& Morris, 2001). Although it is considered a time window of increased vulnerability for mental health problems, adolescence also represents a window of opportunity for interventions that reduce the impact of risk factors and build resilience to future challenges (Collishaw, 2015). One candidate factor, self-compassion, or being kind towards oneself in challenging times (Neff, 2003), has been positively associated with better mental health (MacBeth \& Gumley, 2012) and better social functioning (Neff \& Beretvas, 2013). 
There is emerging evidence that individual differences in self-compassion may be shaped by family experience (Neff \& McGehee, 2010), such as parenting behaviour (Pepping et al., 2015). However, no studies have directly explored the associations between self-compassion, social factors (i.e., parenting behaviour and friendships) and depressive symptoms in adolescents. If a relationship between adolescent depressive symptoms and selfcompassion was demonstrated, it would suggest that its cultivation could be a promising therapeutic target.

Additionally, culture is a critical factor in the development of adolescent psychopathology (Polanczyk et al., 2015). Growing up in an individualistic culture such as the UK that promotes values of personal goals, or growing up in a collectivistic culture such as China that promotes social harmony (Markus \& Kitayama, 1991; Singelis, 1994), could differentially influence how selfrelated characteristics and interpersonal relationships contribute to adolescent depression (Greenberger et al., 2000; Stewart et al., 2004). Understanding of the potential associations between culturally-dependent social factors, such as parenting behaviour and friendships, with self-compassion and depressive symptoms in adolescent samples from different cultures is a current research gap that needs addressing. We proposed the examination of a developmental pathway model (see Fig. 1) in which selfcompassion is shaped by positive parenting, which was defined and assessed as encompassing praise, involvement and good quality supervision, and in turn, influences close friendships that can be associated with youth depressive symptoms. Below we outline the theoretical and empirical rationale for this model.

\section{Self-Compassion, Adolescent Depressive Symptoms and Friendships}

Being kind and compassionate towards oneself in difficult times enables individuals to process unpleasant emotions in a non-judgmental, receptive way, and to acknowledge such emotions as a transitory, common experience shared by all human beings (Neff, 2003). Self-compassion is positively associated with better mental health and lower levels of psychological distress in adolescence (Marsh et al., 2018; Neff \& McGehee, 2010). Given the association between selfcompassion and adaptive emotion regulation under stress (Inwood \& Ferrari, 2018), it is intuitively plausible that there is a beneficial effect of self-compassion on interpersonal functioning in adolescence (Neff et al., 2018). Self-compassion has been associated with increased prosocial behaviour (Marshall et al., 2020), lower physiological responses to social stressors (Bluth et al., 2016), less interpersonal aggression (Barry et al., 2015) and reduced peer victimisation (Jiang et al., 2016). However, none of these studies has focused on the association between self-compassion and the ability to form and maintain close friendships, which in the current study was defined and assessed by fewer conflicts and higher levels of support and intimate disclosure. Given the importance of close friendships in adolescence as a source of happiness (Demir et al., 2007), it is vital to understand such associations.

Kindness, the ability to accept oneself and others even in times of adversity, and a heightened sense of togetherness with others, are key features of self-compassion and prerequisites to form and maintain healthy, close, supportive friendships. This requires adaptive emotion regulation
Fig. 1 Proposed structural model explaining adolescent depression. Note. "+" means positive association and "-" means negative association

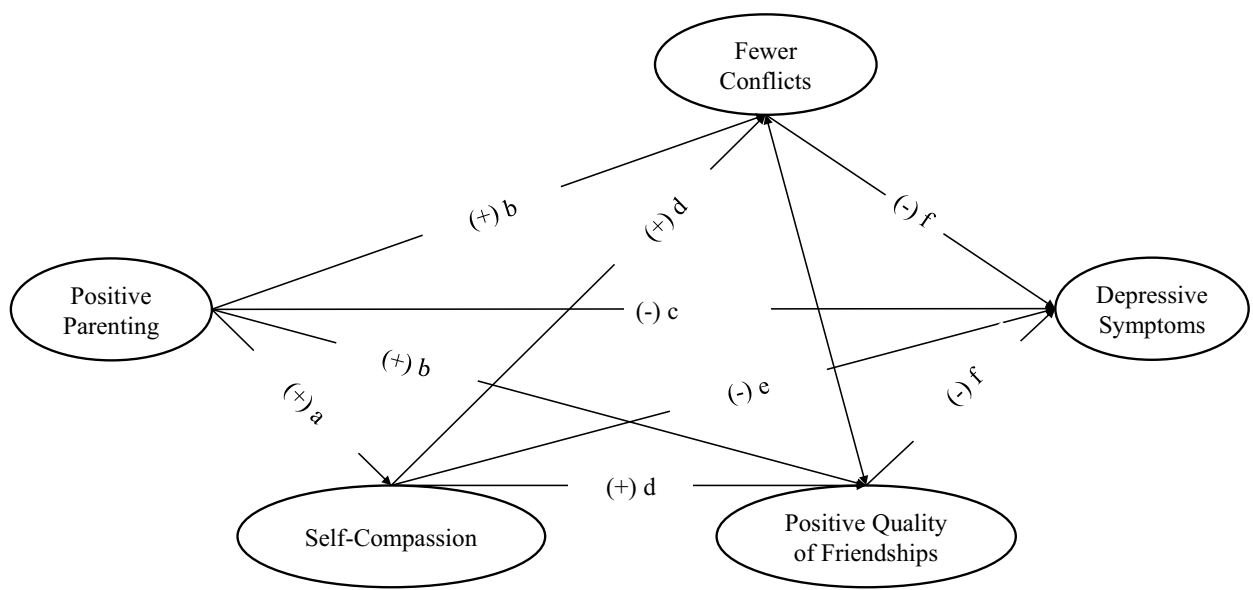

Note. "+" means positive association and "-" means negative association. 
to manage both negative (e.g. conflicts) and positive (e.g., intimacy, support) aspects of relationships (Schwarz et al., 2012) and higher levels of self-compassion have previously been associated with better emotion regulation (Inwood $\&$ Ferrari, 2018). The tripartite model of affect regulation could explain the association between depressive symptoms, self-compassion and friendships (Gilbert, 2005, 2009). When individuals activate their drive or threat system in interpersonal relationships, they are in fight-or-flight mode where they may become aggressive or withdraw from other people. The chronic stress associated with the overactivation of these systems and the related social isolation or lack of social support can contribute to the development of depression (Gilbert, 2009). In contrast, when they activate the soothing system, people tend to feel connected with others, safe and calm (Kirschner et al., 2019). Hence, they could adaptively manage distress and effectively seek and give social support in times of adversity. Therefore, self-compassion should theoretically be associated with better close friendships and fewer depressive symptoms in adolescents (Fig. 1, path d and e).

\section{Positive Parenting Effects on Self-Compassion and Depressive Symptoms}

If self-compassion contributes to adolescent mental health, it is also important to understand developmental social influences that help an individual to develop self-compassion. One candidate, positive parenting, is inversely related to depressive symptoms in adolescence (Smokowski et al., 2015), and depression diagnoses in early adulthood (Costello \& Maughan, 2015). Warm, sensitive and responsive parenting as an individual's early family or caregiver experiences is hypothesised to lead to higher levels of self-compassion (Neff \& McGehee, 2010) which has been supported by previous studies (Pepping et al., 2015; Temel \& Atalay, 2020). We could therefore hypothesise a potential pathway to lower levels of adolescent depressive symptoms from positive parenting via self-compassion (Fig. 1 path a, e, c).

\section{The Potential Pathway to Depressive Symptoms from Parenting Via Friendships}

Based on the interpersonal model of youth depression (Rudolph et al., 2008), maladaptive parenting could also indirectly influence depressive symptoms via poor friendships (Fig. 1, path b c f). Early family disruption is posited to lead to later maladaptive social behaviour, which in turn is more likely to cause problems in interpersonal relationships, such as peer victimisation and low-quality intimate friendships. In turn, such problematic interpersonal relationships undermine adolescents' mental health and predispose to depression. There is empirical evidence supporting aspects of this pathway. Children exposed to high parental psychological control were more likely to struggle to give and receive support, deal with conflict and establish intimacy with their closest friend (Cook \& Fletcher, 2012). Praise and parental involvement were correlated with best friendship qualities (Gaertner et al., 2010). There is also evidence for the friendships-depression pathway of the interpersonal model of youth depression (Rudolph et al., 2008). Negative aspects of best friendships (e.g., conflicts) were associated with higher levels of depressive symptoms (La Greca \& Harrison, 2005) whereas better higher levels of friendship qualities were associated with fewer depressive symptoms (Gaertner et al., 2010) . However, La Greca and Harrison (2005) failed to identify the negative association between positive qualities of friendships and depressive symptoms, and there is also a lack of research directly examining the pathway from parenting to depressive symptoms via friendships.

\section{Self-Compassion as a Potential Mediator between Parenting and Both Friendships and Depressive Symptoms}

The interpersonal model of youth depression (Rudolph et al., 2008) does not include self-related variables. Developing an unhealthy sense of self (e.g., self-criticism, fragile selfesteem) in adolescence may undermine interpersonal relationships and predispose to depressive symptoms. Based on the literature reviewed above, we propose extending the interpersonal model by adding self-compassion as a mediator between parenting, friendships and depression. Selfcompassion in times of adversity is shaped by parenting experiences and in turn may shape the ability to form close friendships and prevent depressive symptoms by allowing individuals to make use of adaptive, positive social support. This developmentally informed sequential path could be understood within the tripartite model of affect regulation (Gilbert, 2005, 2009) and is supported by evidence that self-compassion is shaped by positive parenting (Pepping et al., 2015) and associated with interpersonal functioning (Neff et al., 2018) and lower levels of depressive symptoms (Marsh et al., 2018; Path a, d, c, f in Fig. 1).

\section{Cultural Differences}

Although psychosocial factors are universally important for understanding adolescent depression, when studying it in different countries, it is important to consider cultural differences because culture impacts the socio-emotional and self-identity development (Gibbons \& Poelker, 2019). Specifically, a differential role of individualism versus collectivism has previously been reported in intrapersonal factors, 
interpersonal relationships and mental health (Oyserman et al., 2002).

As proposed in the self-construal theory (Markus \& Kitayama, 1991), individualism promotes the understanding of the self as independent and striving towards personal goals. In contrast, in collectivism, the self is understood as interdependent and inseparable from the social context, and social harmony is prioritised over self-fulfilment. The role of family and friendships in the development of depressive symptoms may, therefore, be more critical in a collectivistic context. For example, the quality of family relationships was found to explain more variance in depressive symptoms in Chinese adolescents than in US adolescents (Greenberger et al., 2000). Conversely, self-related variables such as selfesteem and self-efficacy may be more salient in individualistic societies and hence play a more important role in adolescent mental health than in collective cultures (Choi \& Choi, 2016; Stewart et al., 2004).

However, not all empirical studies on cultural differences in adolescent depression are consistent with the theory. For example, Yamaguchi et al. (2014) found that there is no cultural difference between Japan and the USA regarding the role of self-compassion in depressive symptoms among college students. Neff et al. (2008), although finding cultural differences in levels of self-compassion between college students from Thailand, Taiwan and the USA, revealed no difference when examining the strength of the correlation between self-compassion and depression. Furthermore, Chen et al. (2006) did not find cultural differences in the role of peer harmony and family harmony in adolescents' depressive symptoms between the USA and Hong Kong. These partially mixed findings suggest that further research is necessary to understand potential cultural differences in the way psychosocial factors contribute to adolescent depression. One potential confounder in previous cross-cultural studies is an insufficient understanding of an important methodological issue.

Measurement invariance, this refers to the equivalence of an assessment instrument in different groups (Milfont $\&$ Fischer, 2010) and is an important prerequisite in crosscultural studies to make the findings related to cultural differences or similarities meaningful (Chen, 2008). There is particularly limited evidence of the measurement invariance of self-compassion in certain samples, such as samples from Japan, Taiwan and mainland China for which no measurement invariance could be established (Neff et al., 2008; Tóth-Király \& Neff, 2021). Furthermore, in other empirical studies, there was no explicit consideration of measurement invariance (e.g., Chen et al., 2006; Neff et al., 2008).

The rationale for more cross-cultural research into psychosocial factors for adolescent depression is due to a current paucity of studies focusing on the role of friendships in adolescent depressive symptoms. This is important because of the potentially larger influence of peer relationships on adolescent mental health in collectivistic compared to individualistic cultures (Dekovic et al., 2002). Also, the potential cultural differences could impact on the developmental pathways of young people depressive symptoms which in turn could inform clinical assessment (Polanczyk et al., 2015) and psychological interventions (Herrman et al., 2019). Therefore, this study also aims to examine the assumptions of self-construal theory.

In summary, the current study addresses several gaps in the literature by exploring key assumptions of a theoretically informed path model of positive parenting, self-compassion, friendships and depressive symptoms (Fig. 1). We hypothesised that positive parenting was positively associated with self-compassion (Path a) and friendships (Path b), and associated with fewer depressive symptoms (Path c). Selfcompassion was positively associated with friendships (Path d) and associated with fewer depressive symptoms (Path e). Friendships were associated with fewer depressive symptoms (Path f) in both China and the UK. In line with selfconstrual theory, we expected the coefficients of Path a, b, c and $f$ were bigger in China than the UK, and the coefficients of Path $\mathrm{d}$ and e were bigger in the UK than China.

\section{Method}

\section{Design and Participants}

The theoretical model was assessed using a cross-sectional survey design. The sample consisted of 422 adolescents ( $\mathrm{M}$ age $=14.44, \mathrm{SD}$ age $=1.24 ; 53.0 \%$ female $)$ in the UK and 570 in China ( $\mathrm{M}$ age $=13.41$, SD age $=1.72 ; 43.9 \%$ female), whose age ranged from 11 to 16 years old. The study was approved by the University Ethics Committees (UK).

\section{Measures}

A detailed justification of the choice of measures and procedures to establish its psychometric properties can be found in the supplementary material.

Positive Parenting was assessed using a revised childreport short version of the Alabama Parenting Questionnaire (Scott et al., 2011). The Chinese translation was adapted from the traditional Chinese version of Alabama Parenting Questionnaire by MZ to be appropriate for use in mainland China. Adolescents rated how often a behaviour typically occurs in their home on a five-point Likert scale $(1=$ never; $5=$ always). After a psychometric analysis (Table S1, S2), nine items were used in the final model to represent positive parenting practices. The items were from three subscales; praise (e.g., "Your parents/carers praise you if you behave 
well"), poor supervision (e.g., "You go out with friends your parents/carers don't know") and involvement (e.g., "Your parents/carers help you with your homework"). The internal consistency of the 9-item questionnaire was $\alpha=.82 / .73$, UK/ Chinese Sample.

Self-compassion was assessed using a 12-item version of Stolow's adolescent self-compassion scale (Stolow et al., 2016) and its Chinese translation (Zhao, 2021) in order to reduce assessment burden. Whilst item wording followed the adolescent SCS, the item selection for the short form was informed by Raes' SCS short-form for adults (Raes et al., 2011). Participants were asked to indicate their responses on a 5 -point Likert scale $(1=$ almost never; $5=$ almost always; e.g., "When I fail at something important to me I feel completely stupid"). The psychometric analyses confirmed a general factor of self-compassion, but this construct was different from the original definition (Neff, 2003; Table S5, S6, S7, S8, S9). The internal consistency of self-compassion in both samples was $\alpha=.84 / .76$, UK/ Chinese Sample.

Friendships were assessed via four subscales comprising each three items chosen from the Network of Relationships Inventory (NRI; Furman \& Buhrmester, 1985, 2009). Friendships were measured in relation to the participants' most important friend on a five-point Likert scale (from 1 , little or none, to 5, the most). The three-item conflict subscale assessed negative friendship qualities (e.g., "How often do you and this person get mad at or get in fights with each other"). Nine items assessed positive friendship quality from three subscales; intimate disclosure (e.g., "How often do you tell this person things that you don't want others to know"), seeks safe haven (e.g., "How much does this person turn to you for comfort and support when s/he is troubled about something") and provides secure base (e.g., "How much do you encourage this person to try new things that $\mathrm{s} / \mathrm{he}$ would like to do but is nervous about?"). For the Chinese version, we picked the corresponding items from Liu's (2015) translation. Psychometric evaluation (Table S3, S4) demonstrated good reliability for measuring both the positive $(\alpha=.93 / .84$, UK/Chinese sample) and negative friendship qualities $(\alpha=.89 / .73$, UK/Chinese sample).

Depressive symptoms were measured using the 13-item short form of the Mood and Feelings Questionnaire (Angold et al., 1995; Cheng et al., 2009). Items asked about feelings or behaviours (e.g., "I cried a lot") in the past two weeks on a three-point scale (from 0 , not true, to 2 , true). Psychometric evaluation (Table S10) revealed that the 13 items could be used as a latent variable, depressive symptoms, with high reliability $(\alpha=.92 / .89$, UK/Chinese sample).

\section{Procedure}

We used two methods (Fig. 2) to recruit participants, firstly via an online survey link on social media; and secondly through local schools (one in Somerset, UK and six in Shandong, China). For socioeconomic background, the school in the UK is located in a rural area, in the South West of England and the city where the Chinese schools were recruited is the capital city of Shandong, Jinan. Given the considerable urban-rural gap in China, we recruited three schools in rural areas of Jinan and three schools in urban areas. For school recruitment, headteachers gave consent for school involvement, then the participant information sheet for parents and parent opt-out consent form were given out to students to take to their parents. On the day of the assessment, which took place in school, students who did not return the parental opt-out form were given an information sheet, assent form, questionnaires and debriefing sheet. For online recruitment, an advertisement with the Qualtrics link was posted in parent Facebook groups. Parents read the participant information sheet and consented for their children to take part in the study, at which point they received a link to pass to their child. Young people read the information sheet and provided assent. After answering the questionnaire, adolescents were sent the debriefing sheet.

\section{Data Analysis}

MPlus 8.3 was used for data analysis. Weighted least squares mean and variance adjusted estimation (WLSMV) and the theta parameterisation were used for all analyses because likert-type scales should be treated as ordinal categorical variables (Millsap \& Yun-Tein, 2004; Muthén, 1984). Prior to the analyses, the percentage of missing data was assessed. As it was low $(<3 \%)$, pairwise deletion, the default method of managing missing data in Mplus, was used. Negative items were reverse-scored (e.g., poor supervision).

First, we conducted Confirmatory Factor Analysis (CFA) to confirm the measurement constructs based on the theory. For friendships and parenting, bifactor models with one general factor for positive parenting and positive friendship quality were established. We did not replicate the construct of the short-form self-compassion scale of Raes et al. (2011), so we ran a bifactor exploratory factor analysis (EFA) in the UK sample (more information please see supplementary materials) and found that the bifactor model with three specific factors was the best solution, which was also confirmed by acceptable model fits of CFA in the Chinese sample (Table 1). CFA results supported the one-factor model of conflict (Furman \& Buhrmester, 2009) and depressive symptoms following the original one-factor construct 

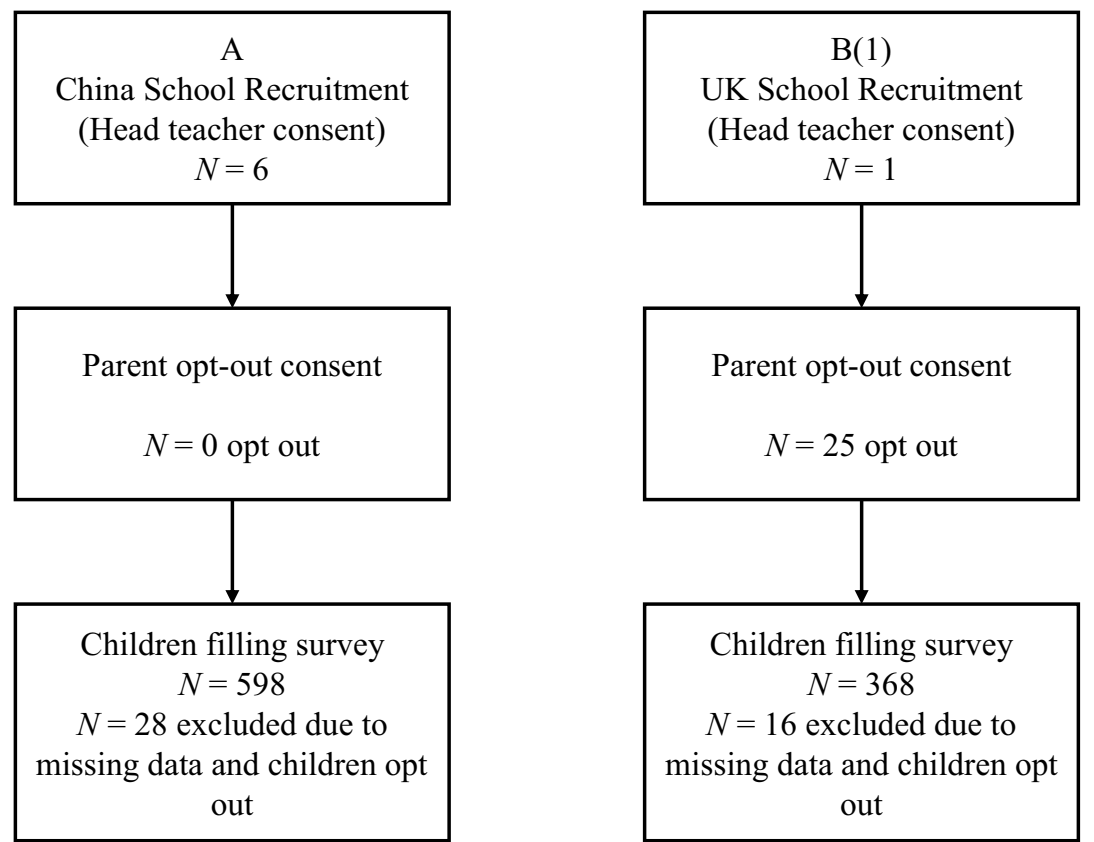

$\mathrm{B}(2)$

UK Online Recruitment (via Facebook social media)

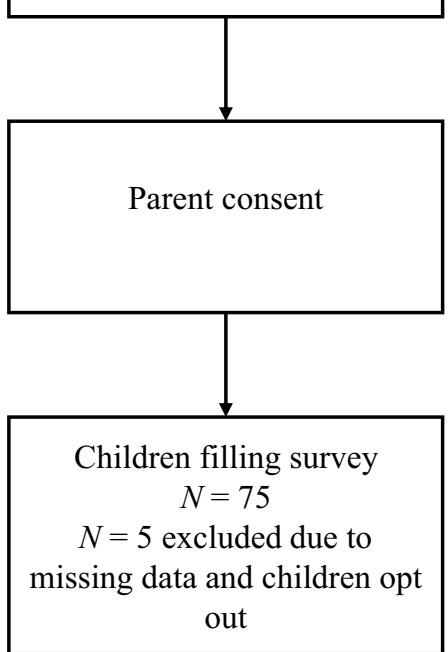

Note. A represents sample recruited via schools in China; $B(1)$ represents sample recruited via school in the UK; $B(2)$ represents sample recruited online in the UK.

Individuals were excluded who had more than $25 \%$ missing data (i.e., children did not answer one scale) and had too consistent answers (for example, if participants always gave the same response) were excluded.

Fig. 2 Participant recruitment flow diagram; procedure for recruitment and data collection. Note. A represents sample recruited via schools in China; $B(1)$ represents sample recruited via school in the $\mathrm{UK} ; B(2)$ represents sample recruited online in the UK. Individu-

(Angold et al., 1995). Based on the CFA results, a structural equation model (SEM) was established to test our hypothesised model. Table 1 shows the model fits of CFA for all measurements in China and UK. The factor loadings of the general factors were all significant. als were excluded who had more than $25 \%$ missing data (i.e., children did not answer one scale) and had too consistent answers (for example, if participants always gave the same response) recruited via school in the UK; $\mathrm{B}(2)$ represents sample

Second, in order to establish the measurement invariance (MI) across samples, a series of nested models with different constraints were conducted via multi-group CFA (Putnick \& Bornstein, 2016). There are different levels of MI, such as configural MI, metric MI and scalar MI. In the current study, the configural and metric invariance across samples were
Table 1 Model fit parameters of CFA results of measreuements in both samples

\begin{tabular}{llllllll}
\hline Measurement & Sample & $\chi^{2}$ & df & CFI & TLI & RMSEA[90\% CI] & SRMR \\
\hline Parenting & UK & 15.584 & 18 & 1.000 & 1.001 & $<.001[.000, .037]$ & .014 \\
& China & $42.630^{* * *}$ & 18 & .995 & .989 & $.049[.030, .068]$ & .026 \\
Positive Friendship Quality & UK & $48.499^{* * *}$ & 18 & .998 & .996 & $.062[.040, .084]$ & .014 \\
& China & $89.028^{* * *}$ & 18 & .982 & .963 & $.083[.066, .101]$ & .026 \\
Conflicts & UK & $<.001^{* * *}$ & 0 & 1.000 & 1.000 & $<.001[.000, .000]$ & .001 \\
& China & $<.001^{* * *}$ & 0 & 1.000 & 1.000 & $<.001[.000, .000]$ & .001 \\
Self-compassion & UK & 53.415 & 38 & .995 & .991 & $.031[.001, .049]$ & .018 \\
& China & $113.368^{* * *}$ & 38 & .967 & .942 & $.059[.047, .072]$ & .029 \\
Depressive symptoms & UK & $207.578^{* * *}$ & 65 & .984 & .981 & $.072[.061, .083]$ & .042 \\
& China & $269.819^{* * *}$ & 65 & .962 & .954 & $.074[.065, .084]$ & .053 \\
\hline
\end{tabular}

CFI Comparative fit index; TLI Tucker Lewis Index; RMSEA Root mean square error of approximation; $C I$ Confidence interval; SRMR Root mean square residual and standardized root mean square residual. ***, $p<.001$ 
examined following recommendations (Putnick \& Bornstein, 2016). For comparing regression coefficients, the MI can be built on the metric level (Chen, 2007). The Chi-square difference statistics and changes in approximate fit indices are presented (Table 2) for the interpretation of the measurement invariance (Sass et al., 2014). We first established configural invariance and then metric invariance. The partial metric invariance was established (Table 2). These analyses revealed partial metric invariance of parenting, friendship, self-compassion and depression was supported, but the metric invariance of conflict was not supported. Specifically, for parenting behaviour, the partial metric invariance of general factors was established except for the loading of one item. For friendship, the partial metric invariance of the general factor was established except for the loading of two items. For self-compassion, the partial metric invariance of the general factor was established except for the loading of four items. For depression, the partial metric invariance of the general factors was established except for the loading of three items. Thus, the differences of association between the general factors of positive parenting, self-compassion, friendships and depressive symptoms could be compared across countries via multi-group SEM, but conflicts could not.

Third, for multi-group SEM, we freed the loadings of some indicators of general factors and all the specific factors, and we also freed the thresholds. The Wald test was used to test for equality of coefficients between countries.

We evaluated the model fits for CFA and SEM based on a joint consideration the value of chi-square/degree of freedom $\left(\chi^{2} / d f, \leq 5\right)$, the values of root mean square error of approximation (RMSEA, $\leq .08$ ), standardised root mean

Table 2 The results of measurement invariance

\begin{tabular}{|c|c|c|c|c|c|c|c|c|c|c|c|}
\hline Model & $\chi^{2}$ & $d f$ & CFI & TLI & $\begin{array}{l}\text { RMSEA [90\% } \\
\mathrm{CI}]\end{array}$ & Model Comparison & $\chi_{\text {Diff }}^{2}$ & $\Delta \mathrm{df}$ & $\Delta \mathrm{CFI}$ & $\Delta$ RMSEA & Decision \\
\hline $\mathrm{M}_{\mathrm{P}_{-} 1}$ & $61.289^{* *}$ & 36 & .997 & .994 & $.038[.020, .053]$ & & & & & & \\
\hline $\mathrm{M}_{\mathrm{P} \_2}$ & $117.490^{* * *}$ & 44 & .992 & .987 & $.058[.045, .071]$ & $\mathrm{M}_{\mathrm{P}_{-} 1}$ vs. $\mathrm{M}_{\mathrm{P}_{2} 2}$ & $38.344^{* * *}$ & 8 & -.005 & -.020 & Reject \\
\hline $\mathrm{M}_{\mathrm{P} \_3}$ & $65.484^{*}$ & 43 & .998 & .996 & $.032[.015, .048]$ & $\mathrm{M}_{\mathrm{P}_{1} 1}$ vs. $\mathrm{M}_{\mathrm{P}_{3} 3}$ & 9.587 & 7 & -.001 & 006 & Accept \\
\hline $\mathrm{M}_{\mathrm{F}_{-} 1}$ & $142.066^{* * *}$ & 36 & .994 & .988 & $.077[.064, .091]$ & & & & & & \\
\hline $\mathrm{M}_{\mathrm{F}-2}$ & $210.784^{* * *}$ & 44 & .991 & .985 & $.088[.076, .985]$ & $\mathrm{M}_{\mathrm{F} \_1}$ vs. $\mathrm{M}_{\mathrm{F} \_2}$ & $62.474^{* * *}$ & 8 & -.003 & -.011 & Reject \\
\hline $\mathrm{M}_{\mathrm{F} \_3}$ & $136.073^{* * *}$ & 43 & .995 & .991 & $.066[.054, .079]$ & $\mathrm{M}_{\mathrm{F}_{-} 1}$ vs. $\mathrm{M}_{\mathrm{F}_{3} 3}$ & $18.754^{* * * *}$ & 7 & .001 & .011 & Reject \\
\hline $\mathrm{M}_{\mathrm{F}_{-} 4}$ & $129.904^{* * *}$ & 42 & .995 & .992 & $.065[.053, .078]$ & $\mathrm{M}_{\mathrm{F}_{-} 1}$ vs. $\mathrm{M}_{\mathrm{F}_{-} 4}$ & 10.870 & 6 & .001 & .012 & Accept \\
\hline $\mathrm{M}_{\mathrm{C}_{-} 1}$ &.$<.001$ & 0 & 1 & 1 & $<.001$ & & & & & & \\
\hline $\mathrm{M}_{\mathrm{C} \_2_{2}}$ & $49.489^{* * *}$ & 2 & .990 & .970 & $.219[.169, .274]$ & $\mathrm{Mc}_{-1}$ vs. $\mathrm{Mc} \_2$ & $49.416^{* * *}$ & 2 & & & Reject \\
\hline Msc_1 & $174.315^{* * *}$ & 76 & .982 & .969 & $.051[.041, .061]$ & & & & & & \\
\hline Msc_2 & $288.185^{* * *}$ & 87 & .963 & .944 & $.068[.060, .077]$ & $\mathrm{M}_{\mathrm{sc} \_1} \mathrm{vs} \mathrm{M}_{\mathrm{sc} \_2}$ & $75.966^{* * * *}$ & 11 & -.019 & .017 & Reject \\
\hline Msc_3 & $228.632^{* * *}$ & 86 & .974 & .960 & $.058[.049, .067]$ & $\mathrm{M}_{\mathrm{sc} \_1} \mathrm{vs} \mathrm{M}_{\mathrm{sc} \_3}$ & $45.082^{* * * *}$ & 10 & -.008 & .007 & Reject \\
\hline Msc_4 & $203.678^{* * *}$ & 85 & .978 & .966 & $.053[.044, .062]$ & $\mathrm{M}_{\mathrm{sc} \_1} \mathrm{vs} \mathrm{M}_{\mathrm{sc} \_4}$ & $31.242^{* * * *}$ & 9 & -.004 & .002 & Reject \\
\hline Msc_5 & $183.446^{* * *}$ & 54 & .982 & .971 & $.049[.039, .058]$ & $\mathrm{M}_{\mathrm{sc} \_1} \mathrm{vs} \mathrm{M}_{\mathrm{sc} \_5}$ & $19.987^{*}$ & 8 & 0 & .003 & Reject \\
\hline Msc_6 & $167.593^{* * *}$ & 83 & .984 & .975 & $.045[.035, .055]$ & $\mathrm{M}_{\mathrm{sc} \_1} \mathrm{vs} \mathrm{M}_{\mathrm{sc} \_6}$ & 9.369 & 7 & .002 & .006 & Accept \\
\hline $\mathrm{M}_{\mathrm{D}_{-} 1}$ & $481.398^{* * *}$ & 130 & .976 & .971 & $.074[.067, .081]$ & & & & & & \\
\hline $\mathrm{M}_{\mathrm{D} \_2}$ & $548.111^{* * *}$ & 142 & .974 & .972 & $.073[.066, .080]$ & $\mathrm{M}_{\mathrm{D} \_1}$ vs $\mathrm{M}_{\mathrm{D} \_2}$ & $71.100^{* * *}$ & 12 & -.002 & -.001 & Reject \\
\hline $\mathrm{M}_{\mathrm{D} \_3}$ & $477.419^{* * *}$ & 141 & .977 & .975 & $.069[.063, .076]$ & $\mathrm{M}_{\mathrm{D} \__{1}}$ vs $\mathrm{M}_{\mathrm{D} \_3}$ & $51.217^{* * *}$ & 11 & -.004 & -.005 & Reject \\
\hline $\mathrm{M}_{\mathrm{D} \_4}$ & $432.478^{* * *}$ & 140 & .980 & .978 & $.065[.058, .072]$ & $\mathrm{M}_{\mathrm{D}_{-} 1}$ vs $\mathrm{M}_{\mathrm{D} \_4}$ & $29.587^{*}$ & 10 & .004 & -.009 & Reject \\
\hline $\mathrm{M}_{\mathrm{D} \_5}$ & $393.733^{* * *}$ & 139 & .983 & .981 & $.061[.054, .068]$ & $\mathrm{M}_{\mathrm{D}_{1} 1}$ vs $\mathrm{M}_{\mathrm{D} \_5}$ & 10.326 & 9 & .007 & -.013 & Accept \\
\hline
\end{tabular}

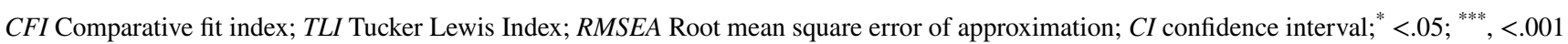

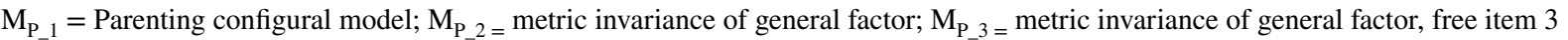

$\mathrm{M}_{\mathrm{F}_{-} 1}=$ Friendships configural model; $\mathrm{M}_{\mathrm{F}_{-} 2}$ = metric invariance of general factor; $\mathrm{M}_{\mathrm{F}_{-} 3}$ = metric invariance of general factor, free item 8; $\mathrm{M}_{\mathrm{F}_{-} 4}=$ metric invariance of general factor, free items 8 and 2;

$\mathrm{M}_{\mathrm{C} \_1}=$ Conflicts configural model; $\mathrm{M}_{\mathrm{C} \_2}=$ metric model;

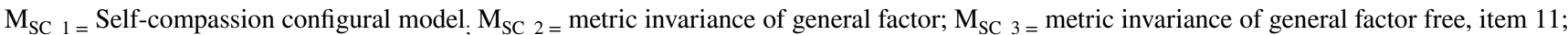
$\mathrm{M}_{\mathrm{SC}_{-} 4=}$ metric invariance of general factor free, items 11 and $10 ; \mathrm{M}_{\mathrm{SC} \_5}$ = metric invariance of general factor free, items 11,10 and $4 ; \mathrm{M}_{\mathrm{SC} \_5}=$ metric invariance of general factor free, items $11,10,4$ and 2;

$\mathrm{M}_{\mathrm{D}_{-} 1=}$ Depressive symptoms configural model; $\mathrm{M}_{\mathrm{D}_{\_} 2=}$ metric invariance of general factor; $\mathrm{M}_{\mathrm{D}_{-} 3=}$ metric invariance of general factor, free item $4 ; \mathrm{M}_{\mathrm{D} \_4}$ = metric invariance of general factor, free items 4 and 3; $\mathrm{M}_{\mathrm{D} \_5}=$ metric invariance of general factor free, items 4,3 and 9 
square residual (SRMR, $\leq .06$ ), comparative fit index (CFI, $\geq .90$ ) and Tucker-Lewis index (TLI, $\geq .90$ ) followed standard recommendations (Hu \& Bentler, 1999; Marsh et al., 2005).

\section{Results}

Descriptive statistics are summarized in Table 3. The proposed model (Fig. 1) fitted both UK and Chinese samples well: $\chi^{2}=1668.770, d f=945, p<.001$, CFI $=.968$, TLI $=.965$, RMSEA $=.043$, SRMR $=.065$ in the UK sample, and in the Chinese samples, $\chi^{2}=1717.567, d f=945, p<$ $.001, \mathrm{CFI}=.943, \mathrm{TLI}=.938, \mathrm{RMSEA}=.038, \mathrm{SRMR}=.059$.

As illustrated by the models in Figure 3, there were five significant paths with the same direction in both samples (from parenting to depressive symptoms, to self-compassion, and to friendships; from self-compassion to depressive symptoms, and from friendships to depressive symptoms).

In the UK sample (Fig. 3), the predicted positive associations between parenting and self-compassion, and parenting and friendships were confirmed; as were the negative associations between positive parenting and depressive symptoms, and between self-compassion and depressive symptoms. Contrary to the hypotheses, positive quality of friendships was positively associated with depressive symptoms, and self-compassion was not associated with the positive quality of friendships but associated with more conflicts. Conflicts were not associated with depressive symptoms. Mediation path analysis revealed a significant indirect effect of parenting on depression via self-compassion, $\beta=-.32, \mathrm{SE}=.05$, $p<.001$, and via friendship, $\beta=.03, \mathrm{SE}=.01, p<.05$, but this is an inconsistent mediation model (MacKinnon et al., 2000), as the direct and indirect effects have different signs.

In the Chinese sample (Fig. 3), we confirmed positive associations between parenting and self-compassion, plus parenting and positive friendship quality, as well as negative associations between parenting and depressive symptoms, plus self-compassion and depressive symptoms. Although self-compassion was not positively associated with the positive quality of friendships, it was positively associated with fewer conflicts, thus partially supporting the hypothesis that self-compassion and friendships are associated. As in the UK sample, positive quality of friendships was positively associated with depressive symptoms and not associated with self-compassion. However, fewer conflicts were negatively associated with depressive symptoms. Mediation analyses revealed significant indirect effects of parenting on depressive symptoms via self-compassion, $\beta=-.30$, SE $=.04, p<.001$, and via friendships, $\beta=.10, \mathrm{SE}=.03, p<$ .001 .However, the indirect effect of parenting on depressive symptoms via friendships was also an inconsistent mediation
Table 3 Correlation and descriptive statistics (The UK sample: $n=422$; The Chinese sample: $n=570$ ).
Fig. 3 Standardised coefficients of paths in SEM in the UK and Chinese samples. Note. The coefficients labelled on the path, $\mathrm{UK} /$ China $;{ }^{*} p<.05,{ }^{* *} p<.01$, ${ }^{* * *} p<.001$

\begin{tabular}{lllllll}
\hline & & UK & & & \\
\cline { 3 - 6 } & Variables & 1 & 2 & 3 & 4 & 5 \\
\hline China & 1 Positive Parenting & - & $.455^{* * *}$ & $.265^{* * *}$ & $.217^{* * *}$ & $-.441^{* * *}$ \\
& 2 Self-compassion & $.560^{* * *}$ & - & .041 & -.039 & $-.832^{* * *}$ \\
& 3 Positive Quality of Friendships & $.387^{* * *}$ & $.148^{* *}$ & - & .023 & .047 \\
& 4 Fewer Conflicts & $.215^{* * *}$ & $.269^{* * *}$ & $.193^{* * *}$ & - & .024 \\
& 5 Depressive Symptoms & $-.582^{* * *}$ & $-.729^{* * *}$ & -.001 & $-.282^{* * *}$ & - \\
\hline
\end{tabular}

${ }^{*} p<.05,{ }^{* *} p<.01,{ }^{* * *} p<.001$

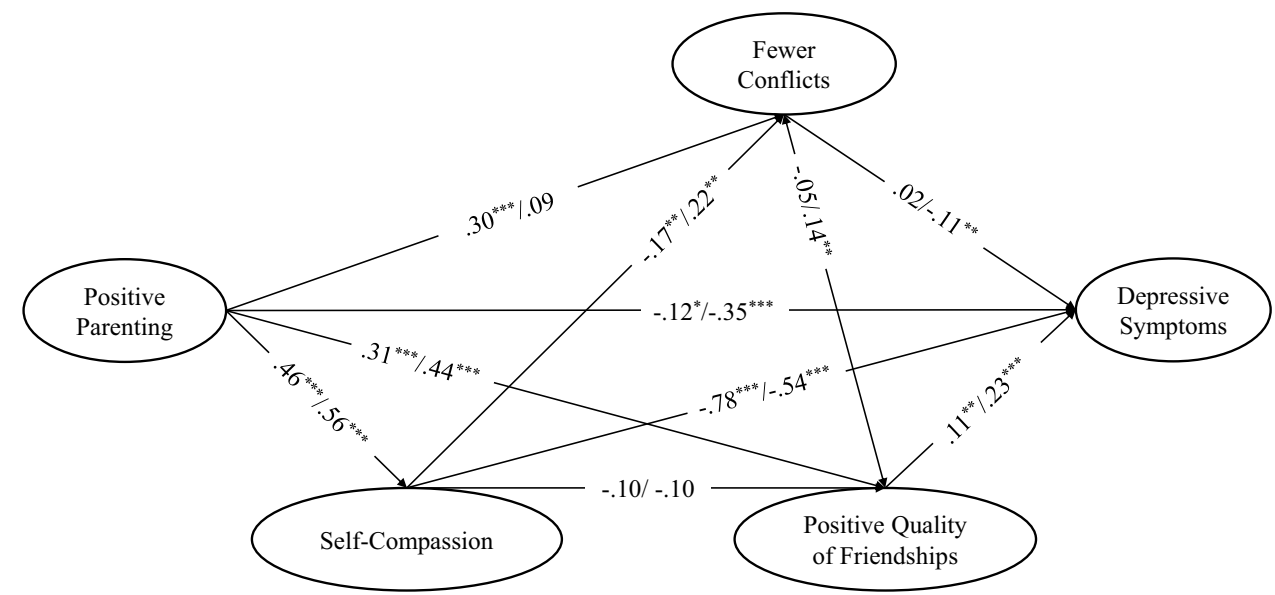

Note. The coefficients labelled on the path, UK/China; ${ }^{*} p<.05,{ }^{* *} p<.01,{ }^{* * *} p<.001$; 
(MacKinnon et al., 2000). Although the association between self-compassion, conflict and depressive symptoms was supported, there was no significant indirect effect of parenting on depression via self-compassion and conflict on depressive symptoms.

Next, we examined differences in path coefficients across countries via multiple-group SEM and Wald test. The model fit for the multigroup SEM was acceptable, $\chi^{2}=3422.703, d f$ $=1919, p<.001, \mathrm{CFI}=.959, \mathrm{TLI}=.956, \mathrm{RMSEA}=.040$, $\mathrm{SRMR}=.063$. Significantly different path coefficients were identified for the paths from parenting to depressive symptoms $\left(\chi^{2}\right.$ (Wald test $\left.)=10.004, \Delta d f=1, p=.002\right)$; from friendships to depressive symptoms $\left(\chi^{2}=5.549\right.$ (Wald test), $\Delta d f=1, p=.019)$; and from parenting to self-compassion $\left(\chi^{2}(\right.$ Wald test $\left.)=8.994, \Delta d f=1, p=.003\right)$. This suggests that the role of interpersonal factors (i.e., parenting and friendships) in self-compassion and depressive symptoms was more salient in the Chinese than the UK sample. In contrast, the association between self-compassion and depressive symptoms was more salient in the UK sample than the Chinses sample, $\chi^{2}$ (Wald test) $=12.102, \Delta d f=1, p<.001$ ). The coefficient of the path from parenting to friendships was marginally significant $\left(\chi^{2}\right.$ (Wald test) $=3.712, \Delta d f=$ $1, p=.054)$.

\section{Discussion}

This cross-sectional study examined a theoretically and empirically informed path model of the associations between parenting, self-compassion and friendships to explain the development of depressive symptoms among adolescents in the UK and China and explored whether cultural differences existed in these relationships. Our hypotheses were partially supported, and although the directions of the associations were broadly similar in the two cultures, there were some significant differences. Contrary to our hypothesis, positive friendships quality was positively associated with depressive symptoms in both countries, and the association between self-compassion and friendships was complex. Self-compassion was associated with fewer conflicts in China but more conflicts in the UK. We demonstrated a path from parenting to depressive symptoms via self-compassion in both countries, but the path via friendships was an inconsistent mediation in both countries, and there was also no strong evidence for a sequential path from parenting to self-compassion to friendships to depressive symptoms in either country.

\section{The Effect of Parenting on Self-Compassion, Friendships and Depressive Symptoms}

Our findings in both samples are consistent with previous research showing that parenting was positively associated with self-compassion (Pepping et al., 2015), better friendship competence (Cook \& Fletcher, 2012) and fewer internalising problems (Smokowski et al., 2015). Theoretically, these findings could be explained by attachment theory, which postulates that early caregiver experiences contribute to the development of an individual's internal working models about the self, others and relationships between self and others (Ainsworth et al., 2015). These models shape how an individual relates to themselves (e.g. "I am lovable") and others (e.g. "I can trust others to be supportive"). Thus, a secure parent-child relationship is thought to exert a positive impact on the ability of the child to acknowledge their own needs, to seek care and compassion and to be able to provide these to others (Neff \& McGehee, 2010; Pepping et al., 2015). This, in turn, reduces the individual's susceptibility to psychopathology (Thompson, 2008). Our findings extend previous research on positive effects of self-compassion on mental health in adolescents (Neff \& McGehee, 2010) by demonstrating for the first time that positive parenting was associated with higher self-compassion which in turn was associated with fewer depressive symptoms, as well as demonstrating a negative association between self-compassion and depressive symptoms.

\section{The Role of Self-Compassion and Friendships in the Path to Depressive Symptoms}

The relationship between self-compassion, friendships and depressive symptoms in the pathway model was equivocal. First, we only partially confirmed the positive association between self-compassion and social functioning in adolescents that has been demonstrated in previous research (Barry et al., 2015; Jiang et al., 2016); this was evident only in the Chinese sample. We based our hypotheses on Gilbert's $(2005,2009)$ theory that when an individual is self-compassionate, the soothing system is activated, which enables adaptive emotion regulation, the ability to self-soothe in stressful situations, to seek support and experience a sense of togetherness with others. If in contrast, the individual is highly self-critical, the threat system is activated, and the individual becomes preoccupied with regulating their distress, which may manifest as anxiety and withdrawal, or anger and aggressive behaviour, thus undermining peer relationships. If the individual seeks pleasure and self-worth by outperforming others, this activates the drive system. A predominant activation of the threat and drive systems is associated with problematic interpersonal relationships and poor mental health (Gilbert, 2014; Taylor et al., 2011). Predominant soothing system activation has been associated with higher wellbeing and more positive social functioning (Gilbert, 2009, 2014; Kirschner et al., 2019). The absence of a significant association between self-compassion and positive friendship qualities in both samples and the 
counterintuitive association between self-compassion and increased conflicts in the UK sample are therefore surprising. We used a short form of the self-compassion scale, and although we established a valid general factor, we did not replicate Neff's (2003) factor structure of self-compassion. To date, there are limited studies exploring the construct of self-compassion in adolescents, and the recently published youth self-compassion scale (Neff et al., 2021) had not been published when we conducted our study. Future studies need to examine in more detail the construct of self-compassion in young people given that during adolescence some core features of self-compassion are still under development and may be harder to assess (Muris et al., 2016).

Contrary to our hypotheses and at odds with theoretical models (Rudolph et al., 2008), we found that positive friendship quality was positively associated with depressive symptoms in both samples. Interestingly, there was no significant zero-order correlation between friendships and depression, and we observed an inconsistent mediation (MacKinnon et al., 2000) in the association between parenting, friendships and depressive symptoms in both cultures (see Table 3 and Fig. 3). This finding is difficult to interpret and requires further investigation in line with Mackinnon et al. (2000) 's recommendation that inconsistent mediation warrants further hypothesis-driven testing and replication in other datasets.

The positive association between positive friendship quality and depressive symptoms was unexpected but is in line with a previous study in male adolescents who reported suicidal thoughts (Kerr et al., 2006). Additionally, Miller et al. (2014) found that positive friendship quality (i.e., companionship, intimacy and satisfaction) at age of 16 was positively associated with adolescent suicidal ideation at age 18. These accumulating findings highlight the importance of a more nuanced exploration of the friendship context, i.e., it could play a beneficial role, but sometimes it may not be protective for mental health (Miller et al., 2014). Interacting with depressed friend in best friendship dyads has been shown to affect the young person by the friend's depressive symptoms, especially for female teenagers (Giletta et al., 2012). This phenomenon is called co-rumination and involves excessively discussing personal problems within a dyadic relationship (Rose, 2002). It has been associated with better self-reported friendship quality but also higher levels of depressive symptoms in adolescents (Spendelow et al., 2017). Additionally, there is evidence that depression travels in social networks (Rosenquist et al., 2011); those who experience higher levels of depression may share similar social networks. Therefore, future longitudinal research into friendship quality and depressive symptoms should consider friendship context.

Additionally, our results are in line La Greca and Harrison (2005) that negative and positive friendships quality might play differential roles in youth depression, suggesting that future research should study these constructs separately. We found that conflicts were associated with more depressive symptoms in our Chinese sample, which could be understood within the youth interpersonal depression model (Rudolph et al., 2008) and is consistent with previous research (La Greca \& Harrison, 2005). The absence of this association in the UK sample is surprising. Measuring friendships quality solely by self-report has previously been criticised by La Greca and Harrison (2005) who recommended the use of other informants as well. Conflicts rated by adolescents may underly social desirability, and this may differ in the two cultures. Therefore, these results require replication and future studies should aim to obtain ratings from another informant about friendships quality.

Altogether, given that self-compassion and friendships are multifaceted concepts (Furman \& Buhrmester, 2009; Neff, 2003) and the psychometric definition of self-compassion requires more research in adolescent samples (Muris et al., 2016), especially in Chinese version (Neff et al., 2019; Tóth-Király \& Neff, 2021), our findings on the association between parenting, self-compassion, friendships and depressive symptoms need replication in a longitudinal design.

\section{Cultural Differences Between China and the UK}

Our findings corroborated cross-cultural theory and previous research, including research on other constructs relating to the self. The stronger association between self-compassion and depressive symptoms detected in the UK compared to the Chinese sample is similar to differences detected by studies comparing the association with self-efficacy or self-esteem and depressive symptoms in these two cultures (e.g., Choi \& Choi, 2016; Stewart et al., 2004). It is also in line with self-construal theory (Markus \& Kitayama, 1991), which suggests that individuals from an individualistic culture are more likely to see themselves as independent, pursue goals of self-fulfilment and value their internal feelings (Singelis, 1994).

However, our findings contrast with previous research which did not detect cultural differences in the association between self-compassion and depressive symptoms (Neff et al., 2008). There were sample differences between these two studies. Neff et al. (2008) included an older sample of young adults, students, aged 19.8 to 21.4 years. Differences in the developmental stages of participants might account for the differences in the findings across these two studies, as in early adolescence self-compassion may still be developing (Muris et al., 2016), but this cannot explain why cultural differences would be observed in early adolescence but not in early adulthood. It is possible that being a student, as the majority of participants in Neff et al. (2008)'s study were, away from the family environment and required to become 
more independent, is a universal, cross-cultural experience, and so may help to explain Neff's findings of no cultural differences in these relationships. Also, Neff et al. (2008) did not report results of measurement invariance tests, which are important in cross-cultural comparisons of the strength of associations (Chen, 2008; Milfont \& Fischer, 2010).

Positive parenting and friendships explained greater amounts of the variance in depressive symptoms among Chinese as compared to UK adolescents, supporting our hypotheses and replicating previous research into cultural differences in the family (Greenberger et al., 2000) and peer relationships (Dekovic et al., 2002). Social context and relationships are essential to the individual in interdependent, collectivistic cultures that strive for social harmony (Markus \& Kitayama, 1991). Cultural differences in parenting behaviour could be rooted in filial piety as a core value in the Chinese culture (Ho, 1996). Perceived parental warmth was positively correlated with children's filial piety, life satisfaction and self-esteem (Leung et al., 2010). Collectivistic culture may sensitise adolescents to interpersonal relationships by the high value it places on the significance of others' feelings and evaluations (Markus \& Kitayama, 1991). The importance of interpersonal factors could be an important consideration for culturally adapted intervention development.

Whereas the cultural differences in the association between parenting and positive friendships were only marginally significant and have not been previously identified, future research should explore this relationship in more depth. Fewer conflicts were negatively related to positive parenting in the UK but not in the Chinese sample where the effect of parenting on conflicts was fully mediated by selfcompassion $(\beta=0.121, \mathrm{SE}=.04, p<.01)$. The path from parenting to conflicts via self-compassion is in line with our hypothesis that, based on attachment theory, internal working models of the self are shaped by caregiver experience, and these internal working models, in turn, affect interpersonal relationships (Ainsworth et al., 2015). This pathway in the Chinese sample is in line with an interdependent self as postulated by the self-construal theory (Markus \& Kitayama, 1991).

The absence of a significant association between parenting and conflicts in the Chinese sample is surprising, as it was observed in the UK sample. Cultural variations in parenting behaviours (Ho et al., 2008; Zhang et al., 2017) may explain these differences. The assessment of parenting behaviour is based on Western conceptualisations of parenting and may have missed behaviours that are more important to parenting in China such as high expectations and strict control (Ho et al., 2008; Zhang et al., 2017), which were not assessed in the current study. Future studies could broaden the measurements to assess these different perspectives. Finally, our results regarding the variable conflicts should be considered with caution because we did not establish measurement invariance.

\section{Strengths and Limitations}

There are several strengths to our study, including its basis in developmental theory with pre-specified hypotheses, reliable and valid measures, and moderately large samples. By establishing the psychometric properties of the measurements and including the measurement constructs in our SEMs, we attempted to reduce measurement errors that could have been induced by using short forms of all scales to reduce assessment burden (Cole \& Preacher, 2014). Additionally, we established measurement invariance across groups (Boer et al., 2018; Milfont \& Fischer, 2010; Stevanovic et al., 2017 ) to ensure reliable and valid measurements that function well across cultures (Stevanovic et al., 2017).

However, there are several limitations which should be noted. We were unable to replicate the self-compassion construct despite using the most appropriate items available to us. There are ongoing psychometric debates about whether a cultural adaption is needed to study self-compassion in Chinese samples (e.g., Neff et al., 2019; Zhao et al., 2021). Although the construct of full version of the self-compassion scale has been replicated in many countries in adults, there are identification problems of the measurement construct in specific cultures, notably China and Japan (Neff et al., 2019; Tóth-Király \& Neff, 2021). The developmental trajectory and profile of self-compassion requires fuller understanding, potentially in tandem with the study of the developmental trajectory of other components of social cognition, such as, empathy, which is important to self-compassion, but is not fully developed in adolescence (Van der Graaff et al., 2014). Future research could use the new youth version self-compassion scale (Neff et al., 2021).

Secondly, it should be cautious about interpreting the cultural difference in the path models. We could not study cultural differences in the role of conflicts across countries because we could not establish measurement invariance for this variable. Conflicts were only assessed by three items and only using self-report. To clarify the association between self-compassion and friendships, it is important to conduct future studies with a clear and replicated construct of selfcompassion and a longer measurement of friendships with multiple informants. For the other variables, we established partial metric measurement invariance. Given the problematic measurement invariance in self-compassion (TóthKirály \& Neff, 2021), future studies could explore and replicate these cultural differences again.

Thirdly, parenting behaviour was assessed in adolescence, whilst our theory was based on attachment theory which stresses early family relationships. We cannot draw conclusions about the direction or temporal order of our 
associations given the cross-sectional design, and future research should use a longitudinal design. The proposed direction of the associations was theoretically informed by an attachment perspective (Ainsworth et al., 2015), but this is not the only possible pathway between psychosocial factors and adolescent depressive symptoms. For example, youth depressive symptoms can erode peer relationships (Kochel et al., 2017) and the sense of oneself (Sowislo \& Orth, 2013). Future longitudinal research should investigate these alternative path models. Furthermore, we are aware of the bias associated with the use of cross-sectional design to address mediation (Maxwell \& Cole, 2007). However, the hypothesized mediations are based on theoretical considerations primarily to understand the amount of variance explained to explore potential theoretical pathways to give tentative evidence for future longitudinal studies.

Fourth, we did not control demographic characteristics (e.g., gender and family socioeconomic status, SES) in SEM as we did not have specific hypotheses for the covariates (Carlson \& Wu, 2011) and lacked the relevant data. Although adolescents are considered able to reliably report depressive symptoms and parenting (Moretti et al., 1985; Parent et al., 2014), the fact that we only used self-report could have led to bias, in particular with respect to reporting friendships. Additionally, because we were only able to acquire self-report measures, we did not assess SES because of known problems such as high levels of invalid and missing data when adolescents are invited to self-report it (Wardle et al., 2002). Future studies could collect data from multiple informants and on more demographic characteristics.

Fifth, all schools were recruited in one geographical area in each country, so our findings may not generalise to other geographic areas. Also, this may limit the generalizability of findings in other cultural backgrounds. In addition, school recruitment and online recruitment are two different approaches, which may cause response differences and result in biases. However, in the current study, we did not find an effect for recruitment method when controlling for it as a covariate in the UK model, suggesting that it did not affect the results.

\section{Implications}

Our findings highlight the importance of the perception of the self in an interpersonal context in adolescent depression and thus support and extend assumptions of the interpersonal model of youth depression (Rudolph et al., 2008). Although the interpersonal model explained the development of youth depression as a function of parental and friendships relationships, it only partially accounts for the role of the self for forming interpersonal relationships as acknowledged in developmental theories such as attachment theory (Ainsworth et al., 2015). We explored the role of self-compassion within the interpersonal model and suggested it could be crucial to understand the contribution of parental and friend relationships to adolescent depressive symptoms. The cultural differences in our study suggest that it is important to consider culture when developing interventions that aim to prevent or treat depression in young people in different contexts. Overall, we concluded that self-compassion could be a useful therapeutic target for interventions designed to treat or prevent adolescent depression in both cultures.

We have not taken the context of modern society into account when discussing parenting and child development. Modern society is characterized as globalised and involves many new forms of communication (e.g., social media) which could exert influence on parenting and child development, as well as culture. With individuals from different countries or cultural backgrounds more closely connected, cultural differences may change (e.g., become smaller). Hence, future research should take into account the opportunities and challenges that modern society presents for adolescents and parents, such as the effects of globalisation, close contact to individuals from diverse and cultural backgrounds and social media.

\section{Conclusions}

These findings provide a preliminary understanding of the association between parenting, self-compassion, friendships and depression in adolescents from two cultures. Our findings suggest that self-compassion may be a useful therapeutic target to improve close friendships as an important aspect of social functioning and mental health among adolescents and that cultural adaptions accounting for interpersonal factors may be required when designing psychological interventions for different contexts or populations.

Supplementary Information The online version contains supplementary material available at https://doi.org/10.1007/s12144-021-02485-x.

Acknowledgements This work was supported by the Research \& Training Support Grant (RTSG) funds, College of Life and Environmental Sciences (CLES), the University of Exeter. The authors gratefully acknowledge Dr Yang Liu for data analysis strategy and checking Mplus Syntax for data analysis in the study. We gratefully acknowledge all researchers providing the relevant scales, Dr Cheryl So, Professor David C. Zuroff, Professor Jianing You and Professor Wyndol Furman. The authors gratefully acknowledge the support regarding school recruitment from Professor Anna Adlam and Dr Jenny Limond, and the collaboration with local schools in the UK and China. The authors gratefully acknowledge Emma Dennie, Dr Latika Ahuja and Naomi Heffer for proofreading the manuscripts and relevant materials.

Authors' Contributions Mengya Zhao: study design, searching questionnaires, data collection, data analysis and interpretation of data, writing the draft, reviewing and editing the draft. 
Tamsin Ford: interpretation of data, writing the draft, reviewing and editing the draft.

Peng Wang: data collection, reviewing and editing the draft.

Anke Karl: study design and data analysis strategy, interpretation of data, writing the draft, reviewing and editing the draft.

Availability of data and analysis syntax All data and data analysis syntax are available upon request from the authors.

\section{Declarations}

Ethics Approval The study was approved by the Psychology Ethics Committee of the University of Exeter (UK).

Disclosure Statement There is no potential conflict between authors and other institutions.

Open Access This article is licensed under a Creative Commons Attribution 4.0 International License, which permits use, sharing, adaptation, distribution and reproduction in any medium or format, as long as you give appropriate credit to the original author(s) and the source, provide a link to the Creative Commons licence, and indicate if changes were made. The images or other third party material in this article are included in the article's Creative Commons licence, unless indicated otherwise in a credit line to the material. If material is not included in the article's Creative Commons licence and your intended use is not permitted by statutory regulation or exceeds the permitted use, you will need to obtain permission directly from the copyright holder. To view a copy of this licence, visit http://creativecommons.org/licenses/by/4.0/.

\section{References}

Ainsworth, M. D. S., Blehar, M. C., Waters, E., \& Wall, S. N. (2015). Patterns of Attachment: A Psychological Study of the Strange Situation (1st ed.). Psychology Press. https://doi.org/10.4324/97802 03758045 .

Angold, A., Costello, E. J., Messer, S. C., \& Pickles, A. (1995). Development of a short questionnaire for use in epidemiological studies of depression in children and adolescents. International Journal of Methods in Psychiatric Research, 5(4), 237-249.

Barry, C. T., Loflin, D. C., \& Doucette, H. (2015). Adolescent selfcompassion: Associations with narcissism, self-esteem, aggression, and internalizing symptoms in at-risk males. Personality and Individual Differences, 77, 118-123. https://doi.org/10.1016/j. paid.2014.12.036

Bluth, K., Roberson, P. N. E., Gaylord, S. A., Faurot, K. R., Grewen, K. M., Arzon, S., \& Girdler, S. S. (2016). Does Self-compassion Protect Adolescents from Stress? Journal of Child and Family Studies, 25(4), 1098-1109. https://doi.org/10.1007/ s10826-015-0307-3

Boer, D., Hanke, K., \& He, J. (2018). On Detecting Systematic Measurement Error in Cross-Cultural Research: A Review and Critical Reflection on Equivalence and Invariance Tests. Journal of Cross-Cultural Psychology, 49(5), 713-734. https://doi.org/10. 1177/0022022117749042

Carlson, K. D., \& Wu, J. (2011). The Illusion of Statistical Control: Control Variable Practice in Management Research. Organizational Research Methods, 15(3), 413-435. https://doi.org/10.1177/ 1094428111428817

Chen, F. F. (2007). Sensitivity of Goodness of Fit Indexes to Lack of Measurement Invariance. Structural Equation Modeling: A
Multidisciplinary Journal, 14(3), 464-504. https://doi.org/10. 1080/10705510701301834

Chen, F. F. (2008). What happens if we compare chopsticks with forks? The impact of making inappropriate comparisons in cross-cultural research. Journal of Personality and Social Psychology, 95(5), 1005-1018. https://doi.org/10.1037/a0013193

Chen, S. X., Chan, W., Bond, M. H., \& Stewart, S. M. (2006). The Effects of Self-Efficacy and Relationship Harmony on Depression Across Cultures: Applying Level-Oriented and Structure-Oriented Analyses. Journal of Cross-Cultural Psychology, 37(6), 643-658. https://doi.org/10.1177/0022022106292075

Cheng, P.-X., Cao, F.-L., \& Su, L.-Y. (2009). Reliability and validity of the Short Mood and Feelings Questionnaire in Chinese adolescents. Chinese Mental Health Journal, 23(1), 60-62. 72.

Choi, E., \& Choi, I. (2016). The associations between body dissatisfaction, body figure, self-esteem, and depressed mood in adolescents in the United States and Korea: A moderated mediation analysis. Journal of Adolescence, 53, 249-259. https://doi.org/10.1016/j. adolescence.2016.10.007

Cole, D. A., \& Preacher, K. J. (2014). Manifest variable path analysis: Potentially serious and misleading consequences due to uncorrected measurement error. Psychological Methods, 19(2), 300315. https://doi.org/10.1037/a0033805

Collishaw, S. (2015). Annual research review: Secular trends in child and adolescent mental health. Journal of Child Psychology and Psychiatry, 56(3), 370-393. https://doi.org/10.1111/jcpp.12372

Cook, E. C., \& Fletcher, A. C. (2012). A process model of parenting and adolescents' friendship competence. Social development (Oxford, England), 21(3), 461-481. https://doi.org/10.1111/j. 1467-9507.2011.00642.x

Costello, E. J., \& Maughan, B. (2015). Annual research review: Optimal outcomes of child and adolescent mental illness. Journal of Child Psychology and Psychiatry, 56(3), 324-341. https://doi.org/ $10.1111 /$ jcpp. 12371

Dekovic, M., Engels, R. C. M. E., Shirai, T., de Kort, G., \& Anker, A. L. (2002). The role of peer relations in adolescent development in two cultures: The Netherlands and Japan. Journal of CrossCultural Psychology, 33(6), 577-595. https://doi.org/10.1177/ 022022102238270

Demir, M., Özdemir, M., \& Weitekamp, L. A. (2007). Looking to happy tomorrows with friends: Best and close friendships as they predict happiness. Journal of Happiness Studies, 8(2), 243-271. https://doi.org/10.1007/s10902-006-9025-2

Furman, W., \& Buhrmester, D. (1985). Children's perceptions of the personal relationships in their social networks. Developmental Psychology, 21(6), 1016-1024. https://doi.org/10.1037/00121649.21.6.1016

Furman, W., \& Buhrmester, D. (2009). The network of relationships inventory: Behavioral systems version. International Journal of Behavioral Development, 33(5), 470-478. https://doi.org/10.1177/ 0165025409342634

Gaertner, A. E., Fite, P. J., \& Colder, C. R. (2010). Parenting and friendship quality as predictors of internalizing and externalizing symptoms in early adolescence. Journal of Child and Family Studies, 19(1), 101-108. https://doi.org/10.1007/s10826-009-9289-3

Gibbons, J. L., \& Poelker, K. E. (2019). Adolescent development in a cross-cultural perspective. In Cross-cultural psychology: Contemporary themes and perspectives, 2nd ed. (pp. 190-215). Wiley Blackwell. https://doi.org/10.1002/9781119519348.ch9.

Gilbert, P. (2005). Compassion: Conceptualisations, research and use in psychotherapy. Routledge.

Gilbert, P. (2009). Introducing compassion-focused therapy. Advances in Psychiatric Treatment, 15(3), 199-208. https://doi.org/10.1192/ apt.bp.107.005264 
Gilbert, P. (2014). The origins and nature of compassion focused therapy. British Journal of Clinical Psychology, 53(1), 6-41. https:// doi.org/10.1111/bjc.12043

Giletta, M., Scholte, R. H., Prinstein, M. J., Engels, R. C., Rabaglietti, E., \& Burk, W. J. (2012). Friendship context matters: examining the domain specificity of alcohol and depression socialization among adolescents. Journal of Abnormal Child Psychology, 40(7), 1027-1043. https://doi.org/10.1007/s10802-012-9625-8

Greenberger, E., Chen, C., Tally, S. R., \& Qi, D. (2000). Family, peer, and individual correlates of depressive symptomatology among U.S. and Chinese adolescents. Journal of Consulting and Clinical Psychology, 68(2), 209-219. https://doi.org/10.1037//0022-006x. 68.2.209

Herrman, H., Kieling, C., McGorry, P., Horton, R., Sargent, J., \& Patel, V. (2019). Reducing the global burden of depression: a LancetWorld Psychiatric Association Commission. Lancet, 393(10189), e42-e43. https://doi.org/10.1016/s0140-6736(18)32408-5

Ho, C., Bluestein, D. N., \& Jenkins, J. M. (2008). Cultural differences in the relationship between parenting and children's behavior. Developmental Psychology, 44(2), 507-522. https://doi.org/10. 1037/0012-1649.44.2.507

Ho, D. Y. F. (1996). Filial piety and its psychological consequences. In The handbook of Chinese psychology. (pp. 155-165). Oxford University Press.

Hu, L.-T., \& Bentler, P. M. (1999). Cutoff criteria for fit indexes in covariance structure analysis: Conventional criteria versus new alternatives. Structural Equation Modeling, 6(1), 1-55. https:// doi.org/10.1080/10705519909540118

Inwood, E., \& Ferrari, M. (2018). Mechanisms of change in the relationship between self-compassion, emotion regulation, and mental health: A systematic review. Applied Psychology. Health and Well-Being, 10(2), 215-235. https://doi.org/10.1111/aphw.12127

Jiang, Y., You, J., Hou, Y., Du, C., Lin, M. P., Zheng, X., \& Ma, C. (2016). Buffering the effects of peer victimization on adolescent non-suicidal self-injury: The role of self-compassion and family cohesion. Journal of Adolescence, 53, 107-115. https://doi.org/ 10.1016/j.adolescence.2016.09.005

Kerr, D. C., Preuss, L. J., \& King, C. A. (2006). Suicidal adolescents' social support from family and peers: gender-specific associations with psychopathology. Journal of Abnormal Child Psychology, 34(1), 103-114. https://doi.org/10.1007/s10802-005-9005-8

Kirschner, H., Kuyken, W., Wright, K., Roberts, H., Brejcha, C., \& Karl, A. (2019). Soothing your heart and feeling connected: A new experimental paradigm to study the benefits of self-compassion. Clinical Psychological Science, 7(3), 545-565. https://doi. org/10.1177/2167702618812438

Kochel, K. P., Bagwell, C. L., Ladd, G. W., \& Rudolph, K. D. (2017). Do positive peer relations mitigate transactions between depressive symptoms and peer victimization in adolescence? Journal of Applied Developmental Psychology, 51, 44-54. https://doi.org/10. 1016/j.appdev.2017.04.003

La Greca, A. M., \& Harrison, H. M. (2005). Adolescent peer relations, friendships, and romantic relationships: do they predict social anxiety and depression? Journal of Clinical Child and Adolescent Psychology, 34(1), 49-61. https://doi.org/10.1207/ s15374424jccp3401_5

Leung, A.N.-M., Wong, S.S.-F., Wong, I.W.-Y., \& McBride-Chang, C. (2010). Filial piety and psychosocial adjustment in Hong Kong Chinese early adolescents. The Journal of Early Adolescence, 30(5), 651-667. https://doi.org/10.1177/0272431609341046

Liu, Q. (2015). The Research of Interactive Qualities in Close Relationship of ENT Hospitalized Adolescent. [Master's thesis, Shandong University]. CNKI. http://220.191.224.148:9001/KCMS/ detail/detail.aspx?filename $=1015381290 . n h \&$ dbcode=CMFD $\&$ dbname $=$ CMFD2016
MacBeth, A., \& Gumley, A. (2012). Exploring compassion: A metaanalysis of the association between self-compassion and psychopathology. Clinical Psychology Review, 32(6), 545-552. https:// doi.org/10.1016/j.cpr.2012.06.003

MacKinnon, D. P., Krull, J. L., \& Lockwood, C. M. (2000). Equivalence of the mediation, confounding and suppression effect. Prevention Science, 1(4), 173-181. https://doi.org/10.1023/A:10265 95011371

Markus, H. R., \& Kitayama, S. (1991). Culture and the self: Implications for cognition, emotion, and motivation. Psychological Review, 98(2), 224-253. https://doi.org/10.1037/0033-295X. 98.2.224

Marsh, H. W., Hau, K.-T., \& Grayson, D. (2005). Goodness of Fit in Structural Equation Models. In Contemporary psychometrics: A festschrift for Roderick P. McDonald. (pp. 275-340). Lawrence Erlbaum Associates Publishers.

Marsh, I. C., Chan, S. W. Y., \& MacBeth, A. (2018). Self-compassion and psychological distress in adolescents-a meta-analysis. Mindfulness (N Y), 9(4), 1011-1027. https://doi.org/10.1007/ s12671-017-0850-7

Marshall, S. L., Ciarrochi, J., Parker, P. D., \& Sahdra, B. K. (2020). Is self-compassion selfish? The development of self-compassion, empathy, and prosocial behavior in adolescence. Journal of Research on Adolescence, 30(Suppl 2), 472-484. https://doi.org/ 10.1111/jora.12492

Maxwell, S. E., \& Cole, D. A. (2007). Bias in cross-sectional analyses of longitudinal mediation. Psychological Methods, 12(1), 23-44. https://doi.org/10.1037/1082-989x.12

Milfont, T. L., \& Fischer, R. (2010). Testing measurement invariance across groups: Applications in cross-cultural research. International Journal of Psychological Research, 3(1), 111-130. https:// doi.org/10.21500/20112084.857

Miller, A. B., Adams, L. M., Esposito-Smythers, C., Thompson, R., \& Proctor, L. J. (2014). Parents and friendships: A longitudinal examination of interpersonal mediators of the relationship between child maltreatment and suicidal ideation. Psychiatry Research, 220(3), 998-1006. https://doi.org/10.1016/j.psychres. 2014.10.009

Millsap, R. E., \& Yun-Tein, J. (2004). Assessing factorial invariance in ordered-categorical measures. Multivariate Behavioral Research, 39(3), 479-515. https://doi.org/10.1207/S15327906M BR3903_4

Moretti, M. M., Fine, S., Haley, G., \& Marriage, K. (1985). Childhood and adolescent depression: Child-report versus parent-report information. Journal of the American Academy of Child Psychiatry, 24(3), 298-302. https://doi.org/10.1016/S0002-7138(09) 61090-6

Muris, P., Meesters, C., Pierik, A., \& de Kock, B. (2016). Good for the self: Self-compassion and other self-related constructs in relation to symptoms of anxiety and depression in non-clinical youths. Journal of Child and Family Studies, 25, 607-617. https://doi. org/10.1007/s10826-015-0235-2

Muthén, B. (1984). A general structural equation model with dichotomous, ordered categorical, and continuous latent variable indicators. Psychometrika, 49(1), 115-132. https://doi.org/10.1007/ BF02294210

Neff, K. D. (2003). The development and validation of a scale to measure self-compassion. Self and Identity, 2(3), 223-250.

Neff, K. D., \& Beretvas, S. N. (2013). The role of self-compassion in romantic relationships. Self and Identity, 12(1), 78-98. https://doi. org/10.1080/15298868.2011.639548

Neff, K. D., Bluth, K., Tóth-Király, I., Davidson, O., Knox, M. C., Williamson, Z., \& Costigan, A. (2021). Development and validation of the self-compassion scale for youth. Journal of Personality Assessment, 103(1), 92-105. https://doi.org/10.1080/00223891. 2020.1729774 
Neff, K. D., Long, P., Knox, M. C., Davidson, O., Kuchar, A., Costigan, A., Williamson, Z., Rohleder, N., Tóth-Király, I., \& Breines, J. G. (2018). The forest and the trees: Examining the association of self-compassion and its positive and negative components with psychological functioning. Self and Identity, 17(6), 627-645. https://doi.org/10.1080/15298868.2018.1436587

Neff, K. D., \& McGehee, P. (2010). Self-compassion and psychological resilience among adolescents and young adults. Self and Identity, 9(3), 225-240. https://doi.org/10.1080/15298860902979307

Neff, K. D., Pisitsungkagarn, K., \& Hsieh, Y.-P. (2008). Self-compassion and self-construal in the United States, Thailand, and Taiwan. Journal of Cross-Cultural Psychology, 39(3), 267-285. https:// doi.org/10.1177/0022022108314544

Neff, K. D., Tóth-Király, I., Yarnell, L. M., Arimitsu, K., Castilho, P., Ghorbani, N., Guo, H. X., Hirsch, J. K., Hupfeld, J., Hutz, C. S., Kotsou, I., Lee, W. K., Montero-Marin, J., Sirois, F. M., de Souza, L. K., Svendsen, J. L., Wilkinson, R. B., \& Mantzios, M. (2019). Examining the factor structure of the self-compassion scale in 20 diverse samples: Support for use of a total score and six subscale scores. Psychological Assessment, 31(1), 27-45. https://doi.org/ 10.1037/pas0000629

Oyserman, D., Coon, H. M., \& Kemmelmeier, M. (2002). Rethinking individualism and collectivism: Evaluation of theoretical assumptions and meta-analyses. Psychological Bulletin, 128(1), 3-72. https://doi.org/10.1037/0033-2909.128.1.3

Parent, J., Forehand, R., Dunbar, J. P., Watson, K. H., Reising, M. M., Seehuus, M., \& Compas, B. E. (2014). Parent and adolescent reports of parenting when a parent has a history of depression: Associations with observations of parenting. Journal of abnormal child psychology, 42(2), 173-183. https://doi.org/10.1007/ s10802-013-9777-1

Pepping, C. A., Davis, P. J., O'Donovan, A., \& Pal, J. (2015). Individual differences in self-compassion: The role of attachment and experiences of parenting in childhood. Self and Identity, 14(1), 104-117. https://doi.org/10.1080/15298868.2014.955050

Polanczyk, G. V., Salum, G. A., Sugaya, L. S., Caye, A., \& Rohde, L. A. (2015). Annual research review: A meta-analysis of the worldwide prevalence of mental disorders in children and adolescents. Journal of Child Psychology and Psychiatry, 56(3), 345-365. https://doi.org/10.1111/jcpp.12381

Putnick, D. L., \& Bornstein, M. H. (2016). Measurement invariance conventions and reporting: The state of the art and future directions for psychological research. Developmental Review, 41, 71-90. https://doi.org/10.1016/j.dr.2016.06.004

Raes, F., Pommier, E., Neff, K. D., \& Van Gucht, D. (2011). Construction and factorial validation of a short form of the self-compassion scale. Clinical Psychology \& Psychotherapy, 18(3), 250-255. https://doi.org/10.1002/cpp.702

Rose, A. J. (2002). Co-rumination in the friendships of girls and boys. Child Development, 73(6), 1830-1843. https://doi.org/10.1111/ 1467-8624.00509

Rosenquist, J. N., Fowler, J. H., \& Christakis, N. A. (2011). Social network determinants of depression. Molecular Psychiatry, 16(3), 273-281. https://doi.org/10.1038/mp.2010.13

Rudolph, K. D., Flynn, M., \& Abaied, J. L. (2008). A developmental perspective on interpersonal theories of youth depression. In Handbook of depression in children and adolescents. (pp. 79-102). The Guilford Press.

Sass, D. A., Schmitt, T. A., \& Marsh, H. W. (2014). Evaluating model fit with ordered categorical data within a measurement invariance framework: A comparison of estimators. Structural Equation Modeling, 21(2), 167-180. https://doi.org/10.1080/10705511. 2014.882658

Schwarz, B., Stutz, M., \& Ledermann, T. (2012). Perceived interparental conflict and early adolescents' friendships: The role of attachment security and emotion regulation. Journal of Youth and Adolescence, 41(9), 1240-1252. https://doi.org/10.1007/ s10964-012-9769-4

Scott, S., Briskman, J., \& Dadds, M. R. (2011). Measuring parenting in community and public health research using brief child and parent reports. Journal of Child and Family Studies, 20(3), 343-352. https://doi.org/10.1007/s10826-010-9398-z

Singelis, T. M. (1994). The measurement of independent and interdependent self-construals. Personality and Social Psychology Bulletin, 20(5), 580-591. https://doi.org/10.1177/0146167294205014

Smokowski, P. R., Bacallao, M. L., Cotter, K. L., \& Evans, C. B. R. (2015). The effects of positive and negative parenting practices on adolescent mental health outcomes in a multicultural sample of rural youth. Child Psychiatry \& Human Development, 46(3), 333-345. https://doi.org/10.1007/s10578-014-0474-2

Sowislo, J. F., \& Orth, U. (2013). Does low self-esteem predict depression and anxiety? A meta-analysis of longitudinal studies. Psychological Bulletin, 139(1), 213-240. https://doi.org/10.1037/ a0028931

Spendelow, J. S., Simonds, L. M., \& Avery, R. E. (2017). The relationship between co-rumination and internalizing problems: A systematic review and meta-analysis. Clinical Psychology \& Psychotherapy, 24(2), 512-527. https://doi.org/10.1002/cpp.2023

Steinberg, L., \& Morris, A. S. (2001). Adolescent development. Annual Review of Psychology, 52, 83-110. https://doi.org/10.1146/annur ev.psych.52.1.83

Stevanovic, D., Jafari, P., Knez, R., Franic, T., Atilola, O., Davidovic, N., Bagheri, Z., \& Lakic, A. (2017). Can we really use available scales for child and adolescent psychopathology across cultures? A systematic review of cross-cultural measurement invariance data. Transcultural Psychiatry, 54(1), 125-152. https://doi.org/ 10.1177/1363461516689215

Stewart, S. M., Kennard, B. D., Lee, P. W., Hughes, C. W., Mayes, T. L., Emslie, G. J., \& Lewinsohn, P. M. (2004). A cross-cultural investigation of cognitions and depressive symptoms in adolescents. Journal of Abnormal Psychology, 113(2), 248-257. https:// doi.org/10.1037/0021-843x.113.2.248

Stolow, D., Zuroff, D. C., Young, J. F., Karlin, R. A., \& Abela, J. R. Z. (2016). A prospective examination of self-compassion as a predictor of depressive symptoms in children and adolescents. Journal of Social and Clinical Psychology, 35(1), 1-20. https://doi.org/ 10.1521/jscp.2016.35.1.1

Taylor, P. J., Gooding, P., Wood, A. M., \& Tarrier, N. (2011). The role of defeat and entrapment in depression, anxiety, and suicide. Psychological Bulletin, 137(3), 391-420. https://doi.org/10.1037/ a0022935

Temel, M., \& Atalay, A. A. (2020). The relationship between perceived maternal parenting and psychological distress: Mediator role of self-compassion. Current Psychology, 39(6), 2203-2210. https:// doi.org/10.1007/s12144-018-9904-9

Thompson, R. A. (2008). Early attachment and later development: Familiar questions, new answers. In Handbook of attachment: Theory, research, and clinical applications, 2nd ed. (pp. 348-365). The Guilford Press.

Tóth-Király, I., \& Neff, K. D. (2021). Is self-compassion universal? Support for the measurement invariance of the self-compassion scale across populations. Assessment, 28(1), 169-185. https://doi. org/10.1177/1073191120926232

Van der Graaff, J., Branje, S., De Wied, M., Hawk, S., Van Lier, P., \& Meeus, W. (2014). Perspective taking and empathic concern in adolescence: gender differences in developmental changes. Developmental Psychology, 50(3), 881-888. https://doi.org/10. 1037/a0034325

Wardle, J., Robb, K., \& Johnson, F. (2002). Assessing socioeconomic status in adolescents: the validity of a home affluence scale. Journal of Epidemiology and Community Health, 56(8), 595-599. https://doi.org/10.1136/jech.56.8.595 
Yamaguchi, A., Kim, M.-S., \& Akutsu, S. (2014). The effects of selfconstruals, self-criticism, and self-compassion on depressive symptoms. Personality and Individual Differences, 68, 65-70. https://doi.org/10.1016/j.paid.2014.03.013

Zhang, W., Wei, X., Ji, L., Chen, L., \& Deater-Deckard, K. (2017). reconsidering parenting in Chinese culture: Subtypes, stability, and change of maternal parenting style during early adolescence. Journal of Youth and Adolescence, 46(5), 1117-1136. https://doi. org/10.1007/s10964-017-0664-x

Zhao, M., Smithson, J., Ford, T., Wang, P., Wong, N. Y. B., \& Karl, A. (2021). Self-compassion in Chinese Young Adults:
Specific Features of the Construct from a Cultural Perspective. Mindfulness, 12(11), 2718-2728. https://doi.org/10.1007/ s12671-021-01734-1.

Zhao, M. (2021). The Role of Psychosocial Factors in Adolescent Depressive Symptoms. [Doctoral Thesis, University of Exeter]. http://hdl.handle.net/10871/126886

Publisher's Note Springer Nature remains neutral with regard to jurisdictional claims in published maps and institutional affiliations. 